\title{
Comparative Study of the Therapeutic Effects of Two Corpus Luteum Support Regimens on Patients with Frozen Embryo Transfer
}

\author{
Zijing Zhang1*, Weigang Liu ${ }^{*}$, Wenyi Lu1 ${ }^{*}$, Shibao Song1* ${ }^{*}$ Jie Pi1,2\# \\ ${ }^{1}$ The Second Clinical Medical College, Yangtze University, Jingzhou, China \\ ${ }^{2}$ Department of Reproductive, Jingzhou Central Hospital, Jingzhou, China \\ Email: "924743172@qq.com
}

How to cite this paper: Zhang, Z.J., Liu, W.G., Lu, W.Y., Song, S.B. and Pi, J. (2019) Comparative Study of the Therapeutic Effects of Two Corpus Luteum Support Regimens on Patients with Frozen Embryo Transfer. Yangtze Medicine, 3, 43-49. https://doi.org/10.4236/ym.2019.31005

Received: December 27, 2018

Accepted: March 19, 2019

Published: March 22, 2019

Copyright $\odot 2019$ by author(s) and Scientific Research Publishing Inc. This work is licensed under the Creative Commons Attribution International License (CC BY 4.0).

http://creativecommons.org/licenses/by/4.0/

\begin{abstract}
Aim: To investigate the efficacy of two corpus luteum support programs in patients with frozen embryo transfer (FET). Methods: A retrospective analysis of the complete clinical data of 340 patients undergoing in vitro fertilization embryo transfer in the Department of Reproductive Medicine, Jingzhou Central Hospital from September 2016 to July 2018. These patients were divided into group A and B according to the luteal support program. We compared the clinical outcomes of the two corpus luteum preparations by comparing the laboratory parameters of the two groups of patients with clinical pregnancy indicators. Results: We found there was no significant difference in the results of general conditions in the two groups of patients, such as infertility age and duration, body mass index (BMI), basal follicle stimulating hormone (FSH), basal luteinizing hormone ( $\mathrm{LH})$, basal estrogen (E2) levels, the endometrial thickness, the number of transplanted high-quality embryos on the day of transplantation and so on $(\mathrm{P}>0.05)$. We found that the implantation rate $(32.55 \%)$, biochemical pregnancy rate $(53.57 \%)$, and clinical pregnancy rate $(51.78 \%)$ in group B were significantly higher than those in group A $(25.26 \%, 35.11 \%$, and $34.66 \%$, respectively) $(\mathrm{P}<0.05)$. We also found there is no significant difference in spontaneous abortion rate $(15.30 \%$ vs $15.87 \%)$ and multiple pregnancy rate $(13.61 \%$ vs $14.28 \%)$ between patients in group A and group B $(\mathrm{P}>0.05)$. Conclusion: We conclude that patients who are scheduled for frozen embryo transfer use progesterone vaginal sustained release capsules combined with luteal progesterone luteal support, have improved clinical pregnancy rates.
\end{abstract}

\section{Keywords}

Frozen Embryo Transfer, Corpus Luteum Support Program, Efficacy Analysis

${ }^{\star}$ These authors contributed equally. 


\section{Introduction}

Since the development of self-assisted reproductive technology, it has indeed solved the fertility problem of many patients. With the development of society, frozen embryo transfer (FET) technology has become an important supplement to in vitro fertilization embryo transfer (IVF-ET) technology and it is important to improve the pregnancy rate of patients [1]. The transplanted embryos are cryopreserved, which can reduce the waste of embryo, increase the chances of transplanting patients, and reduce the financial burden on patients, but the current embryo implantation rate is still only about 32\% [2]. In the FET cycle, there are three types of endometrial preparation, such as natural cycle hormones, replacement cycles, and promotion cycles. Among them, the hormone replacement cycle method is widely used in clinical practice because of its simple operation and good endometrial receptivity. Since the patients themselves cannot produce the corpus luteum during the hormone replacement cycle, the support program of corpus luteum before and after transplantation is a very important link, which is of great significance for improving endometrial receptivity and increasing pregnancy rate. In order to explore the corpus luteum support program to improve the pregnancy rate of freeze-thaw embryo transfer, this study is mainly through retrospective analysis. In order to explore the luteal support program to improve the pregnancy rate of frozen-thawed embryo transfer, this study retrospectively analyzed the efficacy of two corpus luteum support programs in patients undergoing frozen embryo transfer to explore appropriate methods to meet the needs of patients.

\section{Materials and Methods}

\subsection{Case Data}

A retrospective analysis of the clinical data of the freeze-thaw embryo transfer cycle from September 2016 to July 2018 at the Reproductive Center of Jingzhou Central Hospital. The criteria for selecting patients include the age, which no more than 45 years old. The infertility causes of selected patients mainly include pelvic adhesions, fallopian tube obstruction, ovulation disorders, male factors and so on. The remaining frozen-thawed embryos of the selected patient have at least 2 grade II embryos. Patients who were planned to undergo frozen embryo transfer were enrolled on the second day of menstruation for estradiol (E2), luteinizing hormone (LH), and follicle stimulating hormone (FSH). These hormones were at the early follicular stage, and these patients underwent vagina ultrasound examination of the uterus and double attachment results showed no abnormalities. Patients with the following conditions should be excluded. There are other factors affecting embryo implantation, such as obvious uterine deformity, double uterus, endometrial polyps, hydrosalpinx and intrauterine adhesions, etc. Patients with severe internal surgery, such as unexplained vaginal bleeding, liver dysfunction or liver disease, known or likely breast or genital malignancies should also be excluded. Patients with contraindications to sex hor- 
mones, such as acute porphyria, thrombophlebitis, thromboembolic disease, a history of hormone-related thrombophlebitis, thromboembolic disease, cerebral hemorrhage, a related medical history cannot be included in the study. Other exclusion factors include chromosomal abnormalities in couples, repeated graft failures, and patients known to be allergic to progesterone. The subjects were divided into two groups according to the protocol. One group was A. This group of patients was injected intramuscularly with dydrogesterone and progesterone. This group had 230 cycles in total, and the other group was group B. This group used dydrogesterone and progesterone vaginal sustained release gel, this group of patients has 110 cycles. All patients signed an informed consent form and were approved by the ethics committee of the hospital.

\subsection{Endometrial Preparation Review}

Patients enrolled in the group were admitted to the hospital on the second day of menstruation and given oral estradiol valerate (Bujiale). The medication regimen is that the patient takes it orally once a day, taking $2 \mathrm{mg}$ each time and taking it for 4 days. Then changed to $4 \mathrm{mg}$ per oral administration, after taking 4 days, changed to $3 \mathrm{mg}$ peroral each time, twice a day, taking 4 days. On the second day after the drug was used up, the patient underwent vaginal B-ultrasound examination. If the thickness of the endometrium reached $8 \mathrm{~mm}$, the patient was injected with human chorionic gonadotropin (HCG) 4000 U. On the second day after administration, patients in group A were given oral dydrogesterone $20 \mathrm{mg}$ twice a day, and $40 \mathrm{mg}$ of progesterone liquid preparation was administered intramuscularly once a day. We asked group B patients to take oral dydrogesterone $20 \mathrm{mg}$, twice a day and progesterone vaginal sustained-release gel vaginal medication $90 \mathrm{mg}$, once a day. Patients in group A and group B underwent frozen embryo transfer after 3 days of dydrogesterone. The embryos transplanted in the two groups were frozen embryos on the third day of fertilization. The number of transplanted embryos varies from 1 to 3 . These patients continued to receive progesterone 40 - $60 \mathrm{mg}$ once a day after transplantation, and the luteal support treatment lasted for 2 weeks. Two weeks after transplantation, the patient was asked to take blood and check for HCG and progesterone P. If the patient is pregnant, continue to use dydrogesterone and progesterone. If not, stop using the drug. The patient underwent vaginal B-ultrasound after 4 weeks of transplantation. If the gestational sac was seen, it was diagnosed as clinical pregnancy. The pregnant patients changed the dose of dydrogesterone to $20 \mathrm{mg}$ twice a day, if the patient had a threatened abortion such as abdominal pain and vaginal bleeding. The dose of this drug is adjusted according to the condition.

\subsection{Observation Index}

The main indicators were the laboratory and clinical pregnancy indicators of the two groups of patients, including embryo implantation rate, biochemical pregnancy rate, clinical pregnancy rate, spontaneous abortion rate and multiple 
pregnancy rate. The rate of embryo implantation is the ratio of the number of gestational sacs formed after embryo transfer to the total number of transplanted embryos. The biochemical pregnancy rate is the ratio of the number of cycles of biochemical pregnancy to the total number of transplant cycles. The clinical pregnancy rate is the ratio of the number of clinical pregnancy cycles to the total number of transplant cycles. This spontaneous abortion rate refers to the proportion of the number of spontaneous abortion cycles in the three months after transplantation to the total number of clinical pregnancy cycles. The multiple pregnancy rate is the ratio of the number of cycles of multiple embryos to the total number of transplant cycles.

\subsection{Statistical Analysis}

Data analysis was performed using SPSS13.0 statistical software. The measurement data were expressed as $(\mathrm{X} \pm \mathrm{S})$, and the t-test was used for comparison between groups. The count data is expressed as a percentage (\%), and the chi-square test is used for comparison between groups. The difference was statistically significant at $\mathrm{P}<0.05$.

\section{Results}

\subsection{Comparison of the General Situation between the Two Groups of Patients}

We found there was no significant difference in the results of general conditions in the two groups of patients, such as infertility age and duration, body mass index (BMI), basal follicle stimulating hormone (FSH), basal luteinizing hormone (LH), basal estrogen (E2) levels, the endometrial thickness, the number of transplanted high-quality embryos on the day of transplantation and so on $(\mathrm{P}>0.05)$. The two groups were comparable. See Table 1 for details.

\subsection{Comparison of Pregnancy Outcomes between the Two Groups}

The implantation rate, biochemical pregnancy rate and clinical pregnancy rate of group B were significantly higher than those of group A $(\mathrm{P}<0.05)$. There was no significant difference in spontaneous abortion rate and multiple pregnancy rate between the two groups $(\mathrm{P}>0.05)$. See Table 2 for details.

Table 1. Comparison of general conditions between the two groups $(\mathrm{X} \pm \mathrm{S})$.

\begin{tabular}{|c|c|c|c|c|c|c|c|c|c|}
\hline Group & $\begin{array}{l}\text { Number } \\
\text { of cycles }\end{array}$ & $\begin{array}{l}\text { Average } \\
\text { age }\end{array}$ & $\begin{array}{l}\text { Infertility } \\
\text { years }\end{array}$ & $\begin{array}{c}\text { Basic FSH } \\
(\mathrm{U} / \mathrm{L})\end{array}$ & $\begin{array}{l}\text { Basic LH } \\
(\mathrm{U} / \mathrm{L})\end{array}$ & $\begin{array}{l}\text { Basic } \mathrm{E}_{2} \\
(\mathrm{pmol} / \mathrm{L})\end{array}$ & BMI $\left(\mathrm{kg} / \mathrm{m}^{2}\right)$ & $\begin{array}{l}\text { Intima thickness } \\
\text { high quality }(\mathrm{mm})\end{array}$ & $\begin{array}{c}\text { Transplanting } \\
\text { embryos }\end{array}$ \\
\hline Group A & 230 & $29.10 \pm 3.22$ & $3.45 \pm 1.98$ & $6.04 \pm 1.23$ & $7.28 \pm 3.38$ & $185.50 \pm 104.7$ & $24.84 \pm 2.40$ & 9.40 & 0.13 \\
\hline Group B & 110 & $28.99 \pm 3.11^{\#}$ & $3.48 \pm 1.87^{\#}$ & $6.05 \pm 1.30^{\#}$ & $7.38 \pm 3.44^{\#}$ & $174 \pm 88.86^{\#}$ & $25.23 \pm 2.53^{\#}$ & $9.53 \pm 1.49^{\#}$ & $1.97 \pm 0.22^{\#}$ \\
\hline
\end{tabular}

Note: Compared with group A, ${ }^{*} \mathrm{P}>0.05$.

\begin{tabular}{ccccccc}
\hline Group & Number of cycles & Primary infertility & Secondary infertility & Fallopian tube factor & Endometriosis & Ovulation disorder \\
\hline Group A & 230 & 130 & 60 & 100 & 111 & 74 \\
Group B & 110 & 50 & 52 & 107 & 40 \\
\hline
\end{tabular}


Table 2. Comparison of pregnancy outcomes between the two groups (\%).

\begin{tabular}{ccccccc}
\hline Group & Number of cycles & Planting rate & Biochemical pregnancy rate & Clinical pregnancy rate & Natural abortion & Multi-pregnancy rate \\
\hline Group A & 230 & $25.26(118 / 467)$ & $35.11(79 / 225)$ & $34.66(78 / 225)$ & $15.30(15 / 98)$ & $13.61(32 / 235)$ \\
Group B & 110 & $\mathbf{3 2 . 5 5 ( 7 0 / 2 1 5 ) *}$ & $\mathbf{5 3 . 5 7 ( 6 0 / 1 1 2 ) *}$ & $\mathbf{5 1 . 7 8 ( 5 8 / 1 1 2 ) ^ { * }}$ & $15.87(10 / 63)$ & $14.28(16 / 112)$ \\
\hline
\end{tabular}

Note: Compared with group $\mathrm{A},{ }^{*} \mathrm{P}<0.05$.

\section{Discussion}

Under normal circumstances, women who are naturally conceived can detect a certain level of progesterone in the blood after ovulation, which is secreted by the corpus luteum, which can last for one week. The embryo can form a gestational corpus luteum after implantation, and can continue to secrete progesterone to maintain pregnancy [3]. At present, assisted reproductive technology mainly implements frozen embryo transfer. Patients who undergo frozen embryo transfer cannot form endogenous corpus luteum because they do not ovulate. Therefore, external progesterone should be added before and after embryo transfer to transform endometrium and maintain pregnancy until embryo formation. Current progesterone preparations are administered intramuscularly, orally, vaginally, and dermatologically. Progesterone injection is widely used in clinical practice because of its favorable price. However, progesterone is an oily preparation and is not easily absorbed. If the patient has long-term intramuscular injection of oil, it will cause induration, itching, redness and swelling of the skin of the patient, and even more may cause inflammatory reactions such as artificial panniculitis, which are painful for the patient. Oral preparations because of the first-pass effect of the liver, the concentration of the drug in the uterus is low, the efficacy of this preparation is poor, now most patients take the strategy of combining these two medications [4] [5]. The progesterone sustained-release gel is a micronized granule wrapped in a cross-linked polymer, and the curative effect is remarkable and long-lasting, and the capsule is convenient to be administered, and the patient's medication compliance is higher. It has been reported that the progesterone vaginal medication is satisfactory to $89.7 \%$ [6]. However, the corpus luteum vaginal preparation is expensive and the drug residue generated after vaginal administration is accumulated in the vagina of the patient, and it is necessary to perform vaginal washing at intervals, otherwise the drug residue accumulated over a long period of time may adversely affect the patient. Drug residues accumulated by long-term vaginal administration cannot be absorbed, and vaginal washing must be performed regularly. Some patients may be infected with bacteria or mold during vaginal administration. Even patients may cause genital tract infection during pregnancy, retrograde uterine infection, causing threatened abortion, abortion, premature rupture of membranes, premature delivery and other pregnancy complications.

There are advantages and disadvantages of the two drug regimens. Progesterone intramuscular injection is cheaper and more acceptable for infertile patients with poor economic conditions. However, although the progesterone va- 
ginal sustained-release capsule is more expensive, it is convenient to use and has higher comfort. The patient has high medication compliance and the patient can go home to take the medicine.

Studies have found that the vaginal progesterone group has a higher pregnancy rate compared with the intramuscular progesterone group [6], which is consistent with the results of this study. This study found that the implantation rate (32.55\%) and clinical pregnancy rate $(51.78 \%)$ of group B dydrogesterone and progesterone vaginal sustained-release gel group were significantly higher than group A dydrogesterone and progesterone injection group. The embryo implantation rate and clinical pregnancy rate of the progesterone group were $25.26 \%$ and $34.66 \%$ respectively. The results of the two groups were significantly different $(\mathrm{P}<0.05)$. This may be due to the adhesion of the vaginal drug, and the sustained release of the drug may make the drug last longer. The administration of this method avoids the effect of the liver, the blood concentration of the uterus is high after the patient is administered and the side effects of the whole body are also small. However, the biochemical pregnancy rate of vaginal drugs is also high $(53.57 \%$ vs $35.11 \%)$. There are still many shortcomings in this study. For example, only two intimal preparation schemes were selected, which were not included in the natural cycle and microstimulation cycle. The sample size of this study is also small and may result in some bias in the results.

\section{Conclusion}

In summary, progesterone vaginal sustained release gel combined with dydrogesterone for frozen embryo transfer in patients with endometrial preparation can improve the clinical pregnancy rate of patients. This kind of program can relieve the pain of the patient and avoid complications such as artificial induration caused by intramuscular injection of local induration, itching, redness and pain. The using of this medication regimen can also improve the patient's medication compliance, and can also achieve better drug efficacy, with certain clinical guiding value.

\section{Acknowledgements}

We thank Yangtze University for fellowship to Zi-jing Zhang, Wei-gang Liu, Wen-yi Lu and Shi-Bao Song as a graduate student.

\section{Conflicts of Interest}

The authors declare no conflicts of interest regarding the publication of this paper.

\section{References}

[1] (2013) The Effect of Different Luteal Support Programs on Pregnancy Outcomes after Oocyte Maturation Induced by Gonadotropin-Releasing Hormone Agonists in an Antagonist Regimen: A Prospective Randomized Controlled Clinical Trial with Early Termination. Journal of Reproductive Medicine, 22, 797-801. 
[2] Evans, J., Hannan, N.J., Edgell, T.A., et al. (2014) Fresh versus Frozen Embryo Transfer: Backing Clinical Decisions with Scientific and Clinical Evidence. Human Reproduction Update, 20, :808-821. https://doi.org/10.1093/humupd/dmu027

[3] Wang, W.Z. and Zhang, H.W. (2009) The Corpus Luteum Support Method for In Vitro Fertilization-Embryo Transfer Cycle. International Journal of Reproductive Health/Family Planning, 28, 141-144.

[4] Zhang, Y.Z., Wu, Q. and Feng, Y. (2011) Comparison of Corpus Luteum Support for In Vitro Fertilization-Embryo Transfer of Different Drugs. Journal of Tongji University (Medical Sciences), 32, 61-64.

[5] Zheng, B.H., Chen, X.J., Qiu, S.M., et al. (2006) Application of Oral Progesterone in In Vitro Fertilization-Embryo Transfer. Chinese Journal of Practical Gynecology and Obstetrics, 22, 935-936.

[6] Chi, H.B., Zhou, C.Q., Wang, S.Y., et al. (2012) Preliminary Application of Progesterone Vaginal Sustained Release Gel as Corpus Luteum Support in IVF/ICSI Cycle. Journal of Practical Obstetrics and Gynecology, 28, 973-976. 